\title{
Filamentous growth of the budding yeast Saccharomyces cerevisiae induced by overexpression of the WHI2 gene
}

\author{
P. A. Radcliffe, † K. M. Binley, J. Trevethick, M. Hall and P. E. Sudbery \\ Author for correspondence: P. E. Sudbery. Tel: +44 114222 6186. Fax: +44 1142728697. \\ e-mail: P.Sudbery@Sheffield.AC.UK
}

Department of Molecular Biology and Biotechnology, University of Sheffield, Western Bank, Sheffield S10 2TN, UK

\begin{abstract}
The WHI2 gene of the budding yeast Saccharomyces cerevisiae is required for the arrest of cell proliferation upon nutrient exhaustion: whi2 mutants carry on dividing and in the absence of growth become abnormally small. It is reported here that overexpression of Whi2 from the GAL1 promoter results in filamentous growth - cells fail to complete cytokinesis, the budding pattern changes from axial to polar, cells become elongated and cell size increases threefold. In many ways, these filaments resemble the pseudohyphae which result from nitrogen-limited growth and the filaments seen during the invasive growth of haploids. However, Whi2-induced filament formation is reduced, but not blocked, by mutations in STE7, STE12 or STE20 which do block pseudohypha formation. Furthermore, pseudohypha formation can still occur in a diploid in which both copies of the WHI2 gene have been deleted. Thus Whi2-induced filament formation and pseudohypha formation must come about through the action of different pathways. Despite this, a mutation in the STE11 gene, which is required for pseudohypha formation, does block Whi2-induced filament formation. Concanavalin A pulse-chase experiments show that new cell wall material is incorporated only into the tips of the apical cells. An extragenic suppressor of the whi2 allele also results in filamentous growth. These results suggest that Whi2 negatively regulates a function required for the budding mode of cell proliferation including cytokinesis. This function is defined wholly or in part by the fsw1 allele.
\end{abstract}

Keywords: cell cycle, filamentous growth, pseudohyphae, Saccharomyces cerevisiae, WHI2

\section{INTRODUCTION}

When the budding yeast Saccharomyces cerevisiae is starved of nutrients, cells arrest in G1 as unbudded, phase-bright cells and undergo a series of changes that allow them to survive the adverse environmental conditions - they contain elevated levels of carbohydrates such as glycogen and trehalose, they are resistant to environmental stresses such as a $50^{\circ} \mathrm{C}$ heat shock and UV irradiation, and cell wall changes result in resistance to cell-wall-degrading enzymes such as $\beta$-glucuronidase

\footnotetext{
†Present address: Laboratory of Cell Regulation, Imperial Cancer Research Fund, 44 Lincoln's Inn Fields, London WC2A 3PX, UK.

Abbreviations: DAPI, 4',6-diamidino-2-phenylindoyl; FITC, fluorescein isothiocyanate; TPCK, $\mathrm{N}$-tosyl-L-phenylalanine chloromethylketone.
}

or zymolyase (Hartwell, 1974; Pringle \& Hartwell, 1981; Saul et al., 1985; Werner-Washburne et al., 1993). There is no strict coupling between stress resistance and G1 arrest, since slow-growing cells in all phases of the cell cycle acquire stress resistance (Elliott \& Futcher, 1993). Yeast cells may respond in other ways to nutrient limitation. Diploid cells sporulate when growing on an aerobic carbon source such as acetate in the absence of nitrogen. When diploid cells are grown on agar plates containing nitrogen-limiting medium they form pseudohyphae, in which budding switches from a bipolar to a unipolar pattern, cytokinesis is not completed and cell growth becomes highly polarized (Gimeno et al., 1992; Gimeno \& Fink, 1992; Wright et al., 1993). As a result filaments of cells protrude from colonies and invade the agar. This response is considered to be a means by which colonies may forage for new sources of nutrients. 
Haploid cells may also show a similar response known as invasive growth, in which filaments of cells protrude in the agar underneath colonies (Wright et al., 1993; Roberts \& Fink, 1994). Like pseudohyphal growth, invasive growth also involves a switching of the budding pattern, in this case from an axial to a bipolar form. The production of filaments in both pseudohypha formation and invasive growth depends on the functioning of the STE7, STE11, STE12 and STE20 genes which are also elements of the pheromone response pathway (Liu et al., 1993 ; Roberts \& Fink, 1994).

The WHI2 gene is required for cell cycle arrest and the acquisition of stress resistance upon nutrient deprivation (Kelly et al., 1988; Mountain \& Sudbery, 1990a, b; Rahman et al., 1988; Saul et al., 1985; Saul \& Sudbery, 1985; Sudbery et al., 1980). whi2 mutants continue to divide upon carbon exhaustion in a YEPD batch culture and the resulting division without growth results in abnormally small cells which finally arrest at random stages of the cell cycle. They also retain the properties of exponentially growing cells and fail to acquire stress resistance. Transcripts of the G1 cyclins, CLN1 and CLN2, persist upon starvation in a whi2 cell and this is responsible for the inappropriate continuation of cell division (Radcliffe et al., 1997). In this paper we report an involvement of WHI2 in determining whether cells proliferate in a unicellular budding or filamentous mode. We found that overexpressing Whi2 results in filamentous growth. Furthermore, $f s w 1$, which is a second site suppressor of whi2, also causes a similar phenotype. We propose that a function or functions required for the unicellular, budding mode of cell division, including FSW 1 , are negatively regulated by WHI2.

\section{METHODS}

Strains and culture conditions. Strains used in this paper are described in Table 1 . They were routinely cultured in YEPD broth $(1 \%$ Difco Yeast Extract, $2 \%$ Difco peptone, plus $2 \%$ glucose) at $26^{\circ} \mathrm{C}$ with shaking. Plates were solidified by the addition of $2 \%$ Difco agar. YEPGal and YEPR af contained $2 \%$ galactose and $2 \%$ raffinose respectively in place of the glucose. CASGal, CASRaf and CASGlu consist of $2 \%$ Difco Casamino acids, $0.67 \%$ Difco Yeast Nitrogen Base and respectively $2 \%$ galactose, raffinose or glucose. CAS-based media select for the maintenance of a URA3-containing plasmid in a ura3 host as Casamino acids do not contain uracil. Cells transformed with pWHIM5 were routinely induced to form filaments by inoculation from a fresh CASGlu plate into $2.5 \mathrm{ml}$ CASGal in a $50 \mathrm{ml}$ Erlenmeyer flask; filaments were then visible after overnight culture at $26^{\circ} \mathrm{C}$. For Western blot experiments cells were pre-grown in CASR af or CASGal as described in the text and figure legends. For measurements of the percentage of cells showing filament formation cells were grown on CAS-based medium to select for the maintenance of the URA3-based pWHIM5.

Epitope-tagging of Whi2 and the construction of pWHIM3, pWHIM4, pWHIM5 and pWHIM7. The overall strategy was to use YCpmyc111, a yeast centromeric plasmid engineered to facilitate the C-terminal addition of the c-myc epitope, EQKLISEEDLN (Reisdorf $e$ t al., 1993). It contains a multiple cloning site with a $3^{\prime} \mathrm{Smal}$ site followed by the nucleotide sequence encoding the epitope. The following primers were used to amplify the WHI2 gene by PCR : 5'GTAGTCGACACGACCCGAAGGAGGGCAAT (upstream), and 5'ACTCCCGGGTCTGCACCCCAATAACGCTC (downstream). The 3' end of the upstream primer hybridized $505 \mathrm{bp}$ upstream of the ATG. The downstream primer had a $5^{\prime}$ extension which contained a SmaI site and was designed so that when the PCR product was cut with $S m a I$ and ligated to SmaI-cut YCpmyc111, the WHI2 ORF would be fused in-frame at its 3' end to the c-myc epitope. After the PCR product had been cloned into YCpmyc111 the reading frame at the junction was verified by sequencing. Although the whole WHI2 ORF was amplified by PCR, the subsequent cloning was performed so that the WHI2 ORF in the final constructs contained only 328 bp derived from PCR-amplified material, that is from a XhoI site to the end of the ORF. The nucleotide sequence of this DNA was checked by sequencing for mutations introduced by the PCR reaction. Plasmids pWHIM3 and pWHIM4 contained 1307 bp upstream of the ATG as far as a Sall site. Unpublished experiments show that this is sufficient for normal WHI2 expression. The vector for pWHIM3 is the centromeric YCpmyc111. The vector for pWHIM4 is the $2 \mu \mathrm{m}$-based pYES2 (Invitrogen). In pWHIM5 the WHI2 ORF is joined to the GAL1 promoter in pYES2 using a BamHI site $258 \mathrm{bp}$ upstream of the ATG and $10 \mathrm{bp}$ downstream of the normal transcription start site. Both Northern and Western hybridization experiments showed that WHI2 expression in pWHIM5 is under the control of the GAL1 promoter. Plasmid pWHIM5 complemented a WHI2-deleted strain, showing that the epitope does not affect gene function. A triple c-myc cassette cloned into pUC119 (this was constructed by S. Kron and kindly provided by $\mathrm{R}$. Jansen, IMP, Vienna), was employed to create pWHIM7. The cassette was amplified by PCR using primers $5^{\prime}$ TATGCCCGGGTGTTCAAG'TCTTC and 5'ATTGCCCGGGAACAAAAGTTG. The primers were designed so that the product could be digested with $X m a I$ and ligated to XmaI-digested $\mathrm{pWHIM} 3$ to create four tandem copies of the epitope, all of which were in-frame with the WHI2 ORF. The structure of the resulting construct was confirmed by sequencing.

Construction of L5366W containing a homozygous deletion of the WHI2 gene. Haploid segregants of L5366 with complementary mating types were isolated; these were all ura3 since the diploid parent was homozygous for this mutation. They were transformed with a linear DNA fragment consisting of the WHI2 region in which 1420 bp was replaced with the URA3 gene, that is from a BamHI site $258 \mathrm{bp}$ upstream of the start of the WHI2 ORF to a Xhol site $328 \mathrm{bp}$ before the end of the ORF. Ura ${ }^{+}$transformants with complementary mating types were crossed to form diploids. As expected these had a strong whi2 phenotype. The deletion was confirmed by Southern hybridization. The results presented here have been repeated with four independently isolated diploid strains homozygous for the WHI2 deletion.

Detection of epitope-tagged Whi2 by Western blotting. Cells were grown to an $\mathrm{OD}_{600}$ of 0.3 in CASRaf, harvested by centrifugation, resuspended in an equal volume of YEPGal or YEPD and incubated for a further $3 \mathrm{~h}$. Approximately $10^{9}$ cells were harvested by centrifugation, washed in ice-cold water and resuspended in lysis buffer consisting of $50 \mathrm{mM}$ Tris/ $\mathrm{HCl}$ pH $7 \cdot 5,250 \mathrm{mM} \mathrm{NaCl}, 50 \mathrm{mM} \mathrm{NaF}, 5 \mathrm{mM}$ EDTA, $0 \cdot 1 \%$ NP40, $1 \mathrm{mM}$ freshly prepared PMSF, $0.6 \mathrm{mM}$ dimethylaminopurine, $1 \mu \mathrm{g}$ leupeptin $\mathrm{ml}^{-1}, 10 \mu \mathrm{g}$ TPCK $\mathrm{ml}^{-1}, 10 \mu \mathrm{g}$ soybean trypsin inhibitor $\mathrm{ml}^{-1}$ (all protease inhibitors were purchased from Sigma). To this was added an equal volume of acidwashed glass beads $(40$ mesh $\mathrm{BDH})$ and the mixture vortexed five times for $30 \mathrm{~s}$ with $30 \mathrm{~s}$ intervals on ice. Cell lysis was 
Table 1. S. cerevisiae strains used

\begin{tabular}{|c|c|c|}
\hline Strain & Genotype & Source \\
\hline W303a & $\begin{array}{l}\text { a ade } 2-1, \operatorname{trp} 1-1, \text { can } 1-100, \\
\text { leu2-3, his } 3-11, \text { ura3, GAL1 } \\
\text { SSD } 1-1 D\end{array}$ & $\begin{array}{l}\text { M. Tyers, Samuel Lunenfeld } \\
\text { Research Institute, Toronto }\end{array}$ \\
\hline L5366 & $\mathrm{a} / \alpha$ ura $3 /$ ura 3 & H.-U. Mosch \& G. R. Fink \\
\hline L5626 & a $/ \alpha$ ura $3 /$ ura 3 ste $7 /$ ste 7 & H.-U. Mosch \& G. R. Fink \\
\hline L5625 & a/x ura $3 /$ ura 3 ste $11 /$ ste 11 & H.-U. Mosch \& G. R. Fink \\
\hline L5627 & $\mathrm{a} / x$ ura $3 /$ ura 3 ste $12 /$ ste 12 & H.-U. Mosch \& G. R. Fink \\
\hline L5624 & a/x ura $3 /$ ura 3 ste $20 /$ ste 20 & H.-U. Mosch \& G. R. Fink \\
\hline L5366h & a ura3 & This work \\
\hline L5626h* & ura3 ste7 & This work \\
\hline $\mathrm{L} 5625 \mathrm{~h}^{*}$ & ura3 ste11 & This work \\
\hline $\mathrm{L} 5627 \mathrm{~h} *$ & ura3 ste 12 & This work \\
\hline $\mathrm{L} 5624 \mathrm{~h} *$ & ura3 ste 20 & This work \\
\hline L5366W & $\begin{array}{l}\text { a/ } \alpha \Delta \text { whi } 2:: U R A 3 / \Delta \\
\text { whi2::URA3 }\end{array}$ & This work \\
\hline
\end{tabular}

* The mating type of these strains could not be determined because of the respective ste mutations.

confirmed by microscopic examination. The beads and cell debris were pelleted for $2 \mathrm{~min}$ in a microfuge and the supernatant cleared by a further $10 \mathrm{~min}$ centrifugation. Protein content of the sample was determined using a commercial protein assay reagent (Bio-Rad). Forty-five micrograms of the sample was separated on a $7.5 \%$ SDS-polyacrylamide gel using a Bio-Rad Miniprotean II gel tank. The proteins in the gels were transferred to GeneScreen nylon membranes (DuPont) using a Bio-Rad TransBlot apparatus. Ponceau S (Sigma) staining was used to confirm equal loading of the sample in each lane. Blocking reactions, incubation with primary and secondary antibodies and washes were performed as described in the GeneScreen manual. The primary antibody was the c-myc monoclonal antibody, 9E10 (Santa Cruz Biotechnology) diluted 1:100; the secondary antibody was anti-mouse IgG (whole molecule) peroxidase conjugate (Sigma) diluted 1:2000. Immunoreactive material was visualized using enzyme chemiluminescence (ECL; Amersham International).

Cell protein was fractionated into soluble and insoluble fractions as follows. Induced cells were harvested and disrupted with glass beads as described above. The crude mixture of glass beads and cell debris was cleared by passing the suspension through a Pierce \& Warriner disposable polystyrene column containing a sintered glass plug, by centrifuging for $10 \mathrm{~s}$ at $1000 \mathrm{r} . \mathrm{p} . \mathrm{m}$. The filtrate was then centrifuged for $10 \mathrm{~min}$ at 15000 r.p.m. in a microfuge. The supernatant was reserved as the soluble fraction. The pellet was washed twice with $0.5 \mathrm{ml}$ lysis buffer, resuspended in SDS loading buffer containing $10 \mathrm{mM} \beta$-mercaptoethanol, boiled for $5 \mathrm{~min}$, centrifuged for $5 \mathrm{~min}$ and the supernatant retained as the insoluble fraction. Samples of the soluble and insoluble fractions were adjusted so that, when loaded onto an SDS gel as described above, each sample would originate from an equal amount of cells. After Western blotting, staining with Ponceau $S$ revealed that the soluble fraction contained much more material than the insoluble fraction, distributed into a large number of bands; in contrast the insoluble fraction contained a small number of bands.

Cytological methods and microscopy. Immunofluorescence was carried out according to Pringle et al. (1991) using the c- $m y c$ monoclonal antibody 9E10 (Santa Cruz Biotechnology), diluted $1: 100$, as the primary antibody and fluorescein isothiocyanate (FITC) conjugated to rabbit anti-mouse whole molecule IgG (Sigma), diluted $1: 64$, as the secondary antibody. Cells were examined with a Leitz DMRBE microscope connected to a Leica TCS4D confocal scanning module. Although images presented here show fluorescence in one focal plane, images were examined in other planes to confirm that there was no other out of focus fluorescence. Images were recorded as Tiffs.

FITC conjugated to Concanavalin A (Sigma) was used to visualize cell walls as described by Adams \& Pringle (Adams $\&$ Pringle, 1983). One microgram DAPI per millilitre (Sigma) was incorporated into the mounting medium to visualize nuclei. To visualize bud scars cells were fixed for $2 \mathrm{~h}$ at room temperature in $3.7 \%$ formaldehyde, washed twice in distilled water and resuspended in $1 \mathrm{mg}$ Calcofluor-white $\mathrm{ml}^{-1}$ (Sigma). Cells were incubated for $10 \mathrm{~min}$ in the dark, then washed three times in distilled water before viewing. The fluorescent images from Concanavalin A-FITC, DAPI and Calcofluor-white labelled cells were examined using an Olympus $\mathrm{BH} 2$ microscope with a BH2 RFCA reflected light fluorescent light attachment. Images were recorded as Tiffs using a video camera and microcomputer. Colonies growing on plates were examined and photographed using a Nikon Labphot microscope.

Cell volume. The radii of the major $(a)$ and minor $(b)$ axes of at least 40 mother cells were measured. Assuming cells approximate to a prolate spheroid, their volume $(V)$ can be calculated according to the formula $V=4 / 3 \times \pi \times b^{2} \times a$ (Sudbery et al., 1980).

\section{RESULTS}

\section{Epitope-tagged Whi2}

To identify Whi2 and locate it within the cell it was tagged at its C-terminus with a single copy of the c-myc epitope recognized by the $9 E 10$ monoclonal antibody (see Methods). The tagged protein complemented the size defect of a whiz deletion allele $(w h i 2 \Delta)$ in strain 

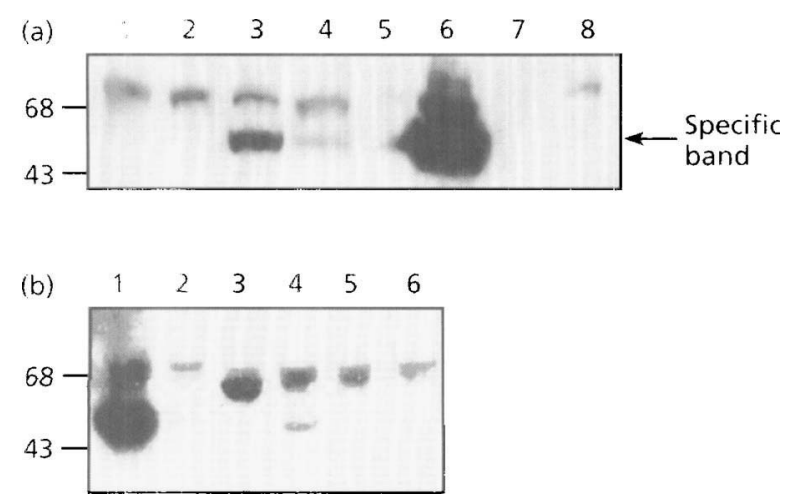

Fig. 1. Overexpression of epitope-tagged Whi2. (a) W303a cells were transformed with pWHIM3 (lanes 1, 2), pWHHIM4 (lanes 3, 4), pWHIM5 (lanes 5, 6) or pYES2 (lanes 7, 8). Cells were pregrown in CASRaf medium then incubated for $3 \mathrm{~h}$ in either YEPD (lanes $1,3,5,7)$ or YEPGal (lanes 2, 4, 6, 8) medium as described in Methods. Proteins were extracted and Western blots prepared using the $9 E 10$ monoclonal antibody. The specific band described in the text is indicated by an arrow. (b) W303a cells were grown overnight on CASRaf and resuspended $\left(\mathrm{OD}_{600}\right.$ $=0.3$ ) in YEPGal medium for $3 \mathrm{~h}$. Lanes: 1 , pWHIM5; 2, pYES; 3 , pWHIM7; 4, pWHIM4; 5, pWHIM3; 6, untransformed. In both parts the molecular mass in $\mathrm{kDa}$ of marker proteins is shown on the left.

W303a (data not shown). The tagged gene also complemented a variety of novel phenotypes which we have recently observed in a W303a whi2s strain hypersensitivity to caffeine, a glycerol growth defect, sensitivity to $1 \mathrm{M} \mathrm{NaCl}$ and a $37{ }^{\circ} \mathrm{C}$ growth defect (unpublished). In fact, in all respects, W303a whi2s cells transformed with plasmids containing the tagged gene had an identical phenotype to the same cells transformed with an equivalent plasmid containing the unmodified gene. Moreover, the effects of overexpressing the tagged gene described below were also observed when the unmodified gene was overexpressed. Thus the epitope tag does appear to interfere with, or modify the function of Whi2.

The tagged gene was incorporated into the following constructs. In pWHIM3 it was expressed from its own promoter on a low-copy centromeric plasmid. In pWHIM4 it was expressed from its own promoter on a multicopy $2 \mu \mathrm{m}$ vector. Finally, in pWHIM5 it was under the control of the GAL1 promoter on a multicopy $2 \mu \mathrm{m}$ vector. Wild-type cells (strain W303a) were transformed with each of the plasmids and Western blots were carried out using protein extracts of cells growing on either galactose or glucose. The results are shown in Fig. 1(a). A number of bands were present in both the negative controls and the experimental samples, these presumably arose by non-specific binding of the monoclonal antibody to proteins present in the total extract. In addition to these non-specific bands, a band of the predicted molecular mass of epitope-tagged Whi2 was evident in pWHIM4-transformed cells growing on galactose or glucose and pWHIM5-transformed cells growing on galactose (indicated by an arrow in Fig. 1a).
The band was absent in pWHIM5-transformed cells growing on glucose and cells transformed with the pYES2 vector. Furthermore, the molecular mass of this band increases by an appropriate amount when four copies of the epitope instead of one are present (see Fig. $1 \mathrm{~b}$ and below). We concluded that this band represents epitope-tagged Whi2. The amount of Whi2 in pWHIM5transformed cells growing on galactose was considerably higher than that from pWHIM4-transformed cells growing on either galactose or glucose.

The level of Whi2 produced by the low-copy pWHIM3 is presumably similar to the normal level of Whi2 in untransformed cells. Since no band was observed in pWHIM3-transformed cells, this level is apparently too low to measure by these means. Because of this we constructed pWHIM7, which consists of a low-copy centromeric vector carrying the WHI2 gene tagged with four copies of the c-myc epitope. The tagged protein was now clearly visible in Western blots (Fig. 1b); indeed the band of immunoreactive material was more intense than that from $\mathrm{pWHIM} 4$, the multicopy vector containing a single copy of the tag. The increased molecular mass of the band is consistent with the extra copies of the c-myc epitope.

\section{Overexpression of WHI2 causes filament formation}

During the course of experiments to detect epitopetagged Whi2 we observed that its overexpression from the strong GAL1 promoter resulted in the formation of filaments, both in liquid culture and on the surface of an agar plate (Fig. 2). The results are described here for strain L5366h but similar results have been obtained for strain W303a (Fig. 6b). L5366h is a haploid strain derived by sporulation from the diploid L5366 kindly provided by H.-U. Mosch \& G. R. Fink, Massachusetts Institute of Technology, MA, USA, as an example of a strain capable of forming pseudohyphae. Normally, L5366h cells grow in liquid culture as spherical clumps of cells due to the failure of cells to separate after cytokinesis; such clumping is common in haploid strains of $S$. cerevisiae. Fig. 2(a) shows pWHIM5-transformed L5366h cells, growing in liquid culture on galactose to induce the expression of Whi2. It can be seen that the cells grew in filaments. Approximately $40 \%$ of cells responded in this way (Table 2 ). Cells transformed with the pYES2 vector had a normal morphology (inset, Fig. 2a). Cell dimensions were measured from this and other similar photomicrographs. The axial ratio (longer axis/ shorter axis) of non-filamenting cells was $1.05 \pm 0.039$, while the axial ratio of filamenting cells was $2 \cdot 27 \pm 0 \cdot 12$. A similar elongation of cell shape occurs during pseudohypha formation in nitrogen-limited diploids and the invasive growth of haploids, although the shape change is less severe in the latter case. Overexpression of Whi2 also results in a three-fold increase in cell volume from $15 \cdot 5$ to $48 \cdot 3 \mu \mathrm{m}^{3}$ (Fig. 2a) showing that overexpression of Whi2 has reset the size control mechanism (Johnston et al., 1977). A whi2 null mutation resets this ratio in the opposite direction (Sudbery et al., 1980); thus Whi2 clearly plays a crucial role in controlling cell size. Most 

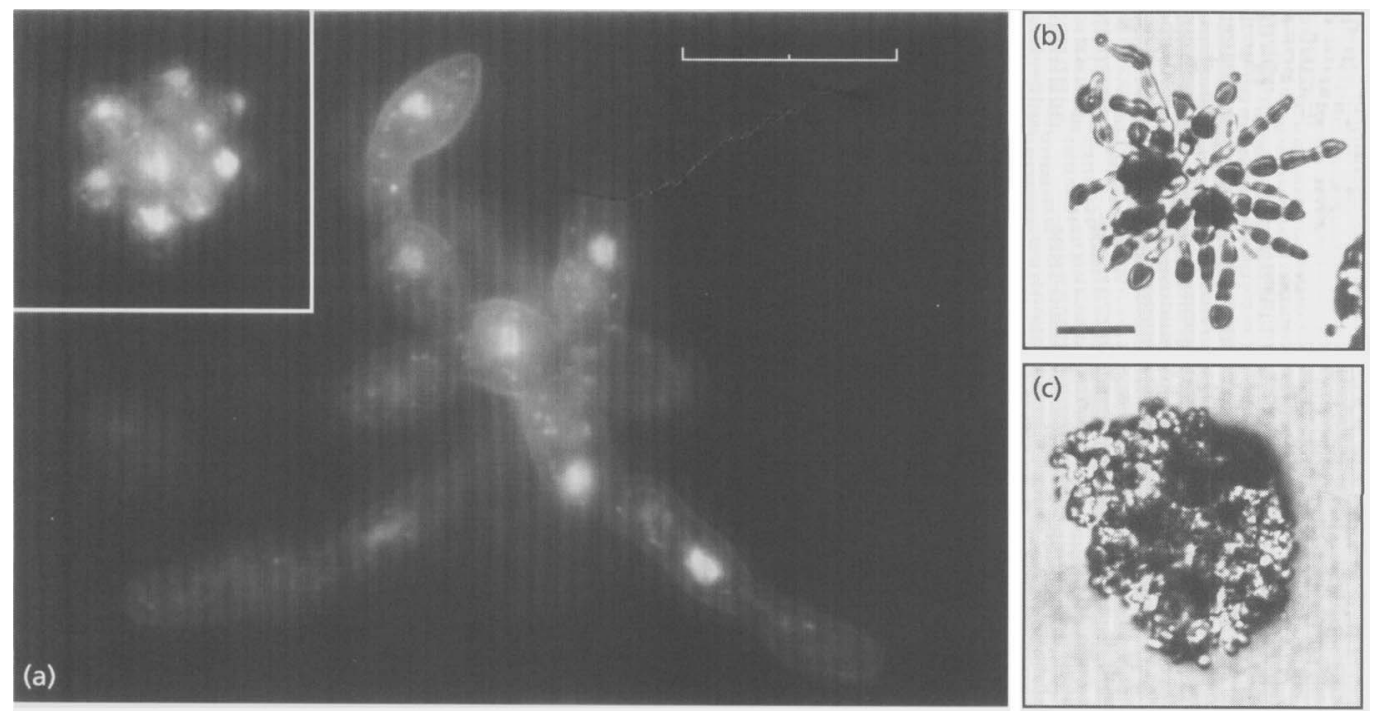

Fig. 2. Effect on cell morphology of Whi2 overexpression. (a) L5366h cells transformed with pWHIM5 (main picture) or pYES2 vector (inset) were grown in liquid CASGal medium, stained with both Concanavalin A-FITC and DAPI and examined with a fluorescence microscope with the filter optimized for DAPI fluorescence. The cell walls are outlined by the residual fluorescence from FITC-Concanavalin A. Note both the main picture and the inset were photographed at the same magnification (bar, $10 \mu \mathrm{m}$ ). L5366h-pWHIM5 cells (b) or L5366h-pYES2 cells (c) were streaked onto the surface of a minimal medium plus galactose agar plate, incubated overnight at $30^{\circ} \mathrm{C}$ and examined by transmitted light microscopy. (b, c) Bar, $20 \mu \mathrm{m}$.

Table 2. Percentage of clumps or microcolonies containing filamenting cells

Cells were cultured overnight on CASGal medium which selects for the presence of the URA3-based pWHIM5 plasmid, harvested, resuspended in fresh CASGal medium to $\mathrm{OD}_{600}=0 \cdot 2$, incubated for a further $4 \mathrm{~h}$ and scored for the percentage of clumps containing filaments; 200 clumps of cells were counted for each value. Generally, either all or none of the cells in a clump showed filament formation; so the presence of a single distinct filament in a clump was used as the criterion to score it as forming a filament. On plates, cells were streaked from a fresh YEPD plate culture onto CasGal, to produce well-separated colonies. After $16 \mathrm{~h}$ incubation the percentage of microcolonies containing filaments was scored in the part of the plate where they were well separated. As in liquid culture, generally either all or none of the cells in a microcolony showed filament formation.

\begin{tabular}{|c|c|c|c|}
\hline Strain & $\begin{array}{l}\text { Relevant } \\
\text { genotype }\end{array}$ & $\begin{array}{c}\text { Filaments on } \\
\text { plates }(\%)\end{array}$ & $\begin{array}{c}\text { Filaments in } \\
\text { liquid culture } \\
(\%)\end{array}$ \\
\hline L5366h-pWHIM5 & WT & $100 \pm 7$ & $40 \pm 3$ \\
\hline L5626h-pWHIM5 & ste 7 & $39 \pm 3$ & $25 \pm 2$ \\
\hline L.5625h-pWHIM5 & ste11 & $0^{-}$ & $0^{-}$ \\
\hline L5627h-p WHIM5 & ste 12 & $36 \pm 3$ & $24 \pm 2$ \\
\hline L5624h-p WHIM5 & ste 20 & $63 \pm 5$ & $20 \pm 2$ \\
\hline
\end{tabular}

of the cells contain nuclei except for the terminal cell in the filament, which is often anucleate; this is presumably the equivalent of a bud still physiologically connected to the adjacent compartment. In addition to nuclei, mitochondria arranged in necklaces are visible around the perimeter of the cells. On plates, filaments form overnight in all of the colonies (Fig. $2 b$ and Table 2). Chains of long thin cells can be seen to radiate from a central clump. After $3 \mathrm{~d}$ the colonies frequently overgrow these extended filaments but, in a sample taken from such colonies, cells can still be seen to be growing in a filamentous fashion. As reported above, overexpression of Whi2 in strain W303a also resulted in filamentous growth both in liquid culture and on plates (see Fig. 6b). This strain does not show the clumping evident in L5366h, and cells normally complete cytokinesis. Thus the failure of cells to separate after cytokinesis, evident in the filaments, represents a real change in the pattern of cell proliferation and is not a result of the tendency of strain L5366h to clump. 

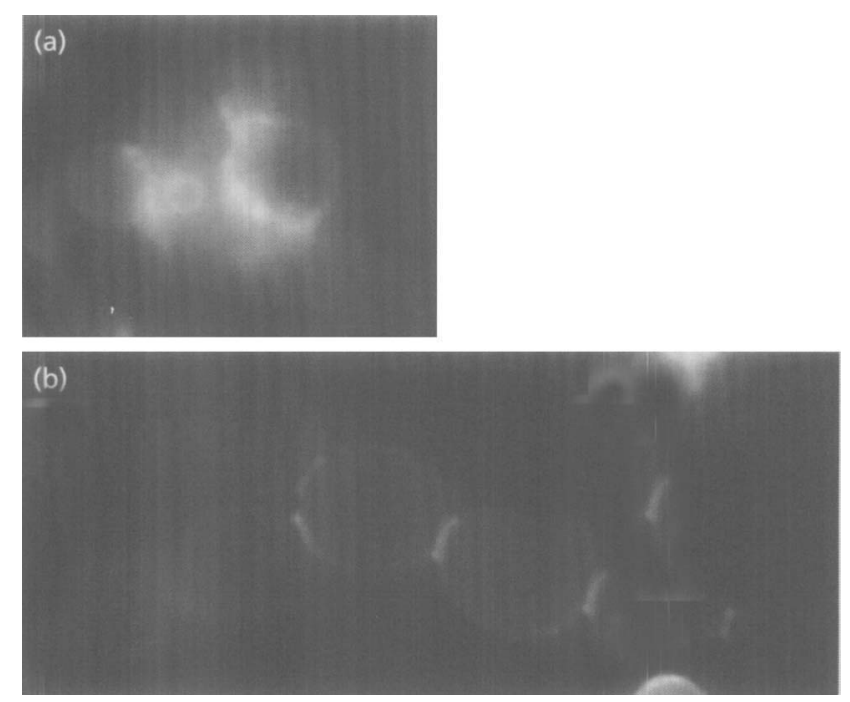

Fig. 3. Bud scar patterns of normal and filamenting L5366h cells. L5366h-pYES2 (a) and L5366h-pWHIM5 (b) cells were grown overnight in YEPGal liquid medium. They were stained with Calcofluor and examined in a fluorescence microscope.

Many of the changes described above are characteristic of cells forming pseudohyphae. However, unlike pseudohyphae, the filamentous growth was not repressed by excess nitrogen, as filaments formed on rich YEP-based, synthetic minimal and nitrogen-limited Sl.AHD (Gimeno et al., 1992) media (data not shown). Cells transformed with pWHIM5 remained viable and formed colonies at a normal rate on galactose. To assess the viability of filamenting cells, individual flaments were transferred by microdissection to a glucose plate. They formed colonies, consisting of normal cells, with a frequency of $100 \%$. The response is specific to Whi2. Cells transformed with the vector alone failed to form filaments [Fig. 2a (inset), c], so it was not an indirect consequence of inducing multiple copies of the GAL1 promoter or of growth on galactose. Nor was the response caused by the c-myc epitope tag since overexpression of untagged Whi2 also caused identical filaments to form (data not shown). Although induced pWHIM5 cells make Whi2 at greatly elevated levels, it does not constitute a significant fraction of total cell protein since no extra band is visible in Coomassiestained gels; thus it is not a non-specific response to protein overexpression. Cells transformed with pWHIM4, which produce more Whi2 than normal cells, but less than pWHIM5-transformed cells on galactose, did not filament on glucose or galactose.

\section{Filament formation results from a change in budding pattern}

Normally, the pattern of budding in a haploid cell is axial; that is, the site of bud formation in a mother cell is adjacent to the site of bud formation in the previous generation or, in a virgin mother cell, at the birth pole of the cell (Freifelder, 1960; Gimeno \& Fink, 1992). If cells fail to separate after cytokinesis, axial budding will result in clumps of cells. Filamentous growth requires both a failure of cells to separate after cytokinesis and a polar pattern of budding, where the daughter bud forms at the free end opposite the birth pole, resulting in linear chains of cells. Calcofluor-staining of non-filamenting cells shows that the bud scars are clustered together in a pattern typical of axial budding (Fig. 3a). Filamenting cells are arranged linearly, with a single bud scar at each junction between the cells, which is clearly not an axial pattern (Fig. 3b). On plates, branches formed two or more cells away from the apical cell and invariably extended in the same direction as the main filament, indicating that the pattern of budding is unipolar; that is, the second and subsequent buds are also restricted to the non-birth pole.

\section{Filament formation is blocked by a mutation in the STE11 gene but not the STE7, STE12 or STE20 genes}

Pseudohypha formation in diploid cells in response to nitrogen-limited growth and the invasive growth of haploids have previously been shown to depend on the functioning of the STE7, STE11, STE12 and STE20 genes (Liu et al., 1993; Roberts \& Fink, 1994). These genes were first described as elements of the pheromoneresponse pathway (for a review see Kurjan, 1993). Haploid strains containing disruptions in these genes (derived from diploids provided by H.-U. Mosch \& G. R. Fink) were tested to see if they still produced filaments in response to Whi2 overproduction. A mutation in the STE11 gene completely abolished the filamenting response both in liquid culture and on the surface of an agar plate (Table 2). However, in ste 7 , ste 12 or ste 20 mutants, filaments formed which were similar in appearance to those that occur in wild-type cells, although the frequency of filamenting cells was reduced (Table 2 ). It should be noted that Table 2 shows that different ste mutants appear to have a different residual capacity for filament formation on plates but apparently equal capacity in liquid culture. Since the function of STE7, STE12 and STE20 is required for pseudohypha formation we conclude that Whi2 operates through a different pathway. This conclusion was confirmed by the observation that a diploid strain, in which both copies of the WHI2 have been deleted (see Methods), still forms pseudohyphae in response to nitrogen-limited growth (Fig. 4). Thus pseudohypha formation does not require WHI2 function.

\section{The filaments show strongly polarized growth}

The elongated appearance of the cells suggested that they were growing in a polarized fashion. To determine if this was so, the Concanavalin A procedure described by Adams \& Pringle (1983) was employed. Cells were grown in YEPGal to allow filaments to form. They were then resuspended in FITC-Concanavalin A to allow existing cell walls to be labelled, the cells were then harvested by centrifugation, washed and resuspended in 


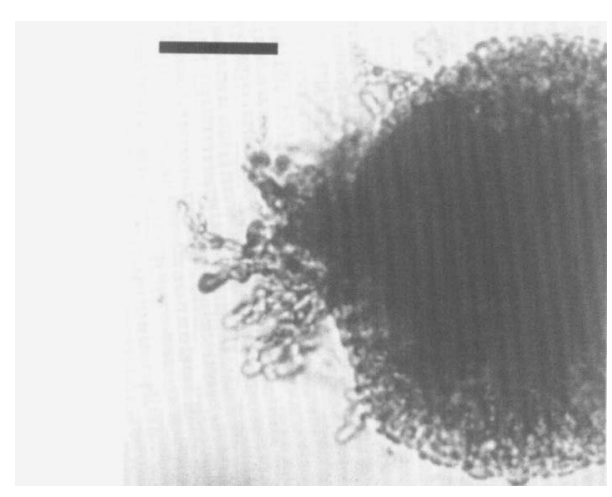

Fig. 4. Pseudohypha formation does not require the function of the WHI2 gene. A diploid homozygous for a WHI2 deletion was cultured for three days on the surface of a SLAHD plate (Gimeno et al., 1992). Bar, $80 \mu \mathrm{m}$.

YEPGal in the absence of FITC-Concanavalin A. After $3 \mathrm{~h}$ they were harvested and the extent of labelled and unlabelled cell wall material determined by fluorescence microscopy. Unlabelled material, representing new growth, occurred exclusively at the tips of the filaments (Fig. 5). This result shows growth of the filaments is strongly polarized, consistent with the elongated appearance of the cells.

\section{When overexpressed, Whi2 is concentrated at the tips of the filaments}

Experiments were carried out to locate epitope-tagged Whi2. No signal was detected in cells transformed with the low-copy plasmids pWHIM3 or pWHIM7, despite the latter containing four tandem copies of the tag which allowed it to be visualized in Western blots. Clearly, in normal cells Whi2 is present in very small amounts. Whi2 was detected when pWHIM5-transformed cells growing on raffinose were induced by the addition of galactose. Five hours after induction it was observed only in the apical cells of the resulting filaments (Fig. 6). When cells were grown for prolonged periods in galactose, some filaments became uniformly labelled, but we never observed filaments in which the internal cells, but not the apical cells, were labelled. In such cultures, not all of the cells show the filamenting response (see above); some, but not all, of the remaining cells showed a positive signal. In cells that were labelled, the protein appeared to be uniformly located throughout the cell. These observations should be interpreted with caution, since it is possible that a specific location was being masked by overproduction of Whi2. No signal was seen in untransformed cells or pWHIM5-transformed cells growing on glucose (data not shown).

Since Whi2 could be detected from the low-copy pWHIM7 in Western blots, but its location not resolved by immunofluorescence, we carried out a simple fractionation of PWHIM7-transformed cells (see Methods). The immunoreactive material was strongly concentrated in the insoluble fraction (Fig. 7).

\section{An extragenic suppressor of whi2 also forms filaments.}

We shall describe elsewhere that whi2 ssd1-d strains have a number of characteristics that suggest cytoskeletal defects (unpublished) including hypersensitivity to caffeine. Growth is inhibited when patched onto plates containing $8 \mathrm{mM}$ caffeine; however papillae of clones carrying a suppressor are evident. Many of these clones show a phenotype which is very similar to that seen on overexpression of WHI2. One such clone has been characterized and shown to be due to a single recessive nuclear mutation which we designate $f s w 1$ (filamenting suppressor of whi2). The appearance of fsw1 cells is shown in Fig. 8. We argue below that the $f s w 1$ allele identifies a downstream, negatively regulated target of WHI2.

\section{DISCUSSION}

Overexpression of Whi2 results in a concerted reorganization of the cell cycle. The budding pattern changes from axial to polar, cytokinesis fails to proceed to completion, cells show hyperpolarized growth, new cell wall synthesis is confined to apical cells, cells become elongated in shape - the axial ratio doubles, and volume increases threefold. The change is reversible: isolated filaments when placed on glucose can act as founders of colonies of yeast-like cells. Taken together these results suggest that a switch to an alternative form of cell proliferation has occurred. These changes could be a non-specific pathology brought about by the effect of overexpressing a gene normally present in low abundance. There a two reasons arguing that this is not the case. Firstly, the level of overexpression is still too low for detection on a Coomassie-Blue-stained gel. Thus the results of overexpression must be a consequence of the specific properties of Whi2 and not due to a general burden on the cell arising out of unbalanced protein synthesis. Perhaps more importantly, a suppressor of the caffeine sensitivity of a whi2 ssd1-d strain has a filamenting phenotype which is very similar to that seen upon WHI2 overexpression. We suggest that the simplest explanation for these observations is that, directly or indirectly, WHI2 negatively regulates a function required for the correct completion of cytokinesis encoded by the FSW1 gene.

This reorganization of the cell cycle described above is also a central feature of pseudohyphal development. However, there are three important differences. Firstly, diploids homozygous for a deletion of the WHI2 gene still form pseudohyphae in response to nitrogen limited growth suggesting that Whi2-induced filament formation acts through a different pathway. Secondly, Whi2induced filament formation does not result in cells invading the agar. Thirdly, Whi2-induced filament formation is not blocked in ste 7 , ste 12 or ste 20 mutants, although it is reduced. The filamenting response is blocked by an ste11 mutation. STE11 encodes a protein kinase homologous to mammalian MEK kinases and to the BCK1 component of the PKC1/MPK1 pathway. A 

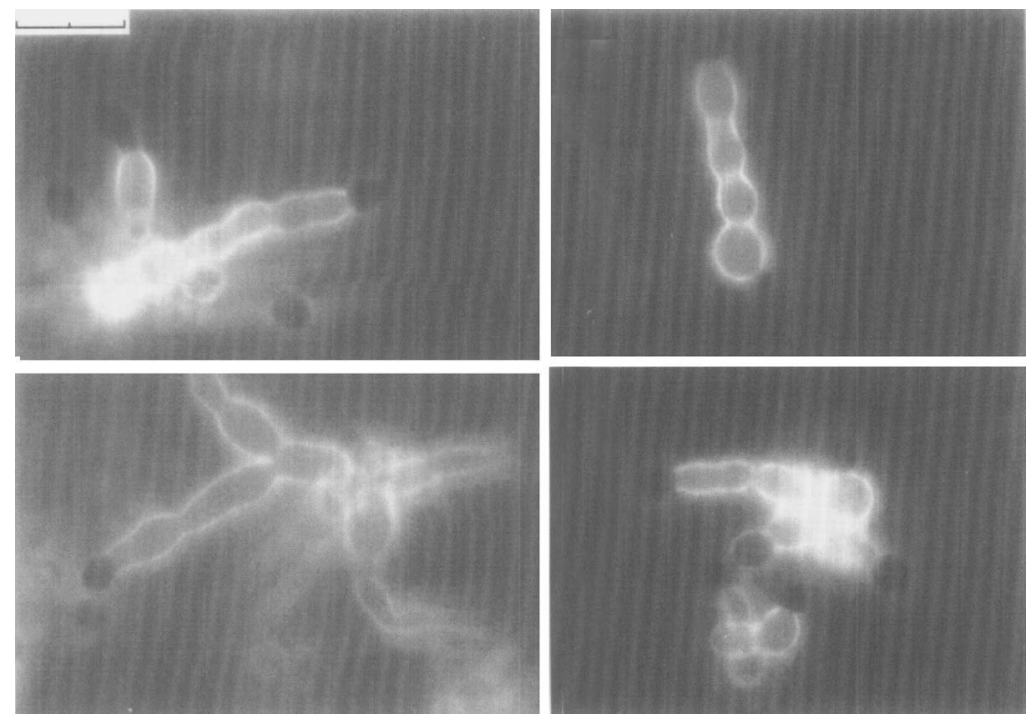

Fig. 5. New cell wall material is incorporated only into the tips of filaments. A Concanavalin A pulse-chase experiment was carried out as described in the text. The figure shows a selection of filaments after the end of the chase period. The dark region at the end of each filament shows new cell material incorporated during the chase period. Bar, $10 \mu \mathrm{m}$.
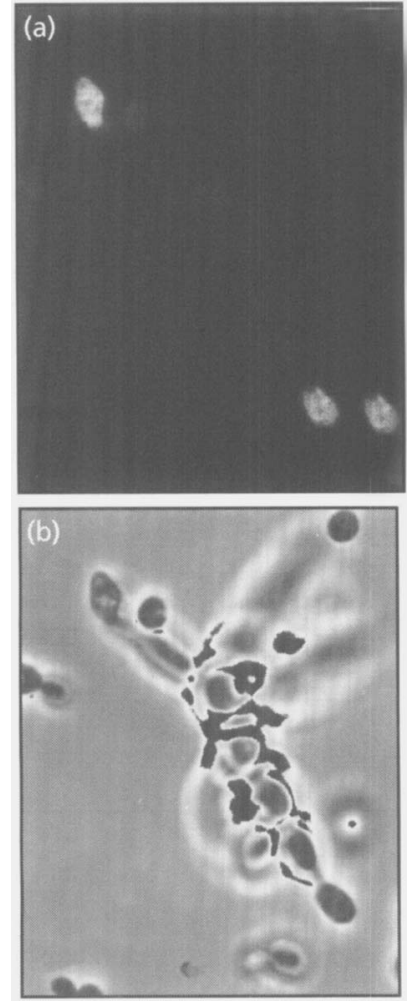

Fig. 6. Epitope-tagged Whi2 is concentrated in the apical cells of the filaments. W303a-pWHIM5 cells were grown to an $\mathrm{OD}_{600}$ of 0.6 on YEPRaf; they were harvested by centrifugation and resuspended in YEPGal and incubated for $5 \mathrm{~h}$. Immunofluorescence was carried out as described in Methods: (a) confocal fluorescence image, (b) confocal phase-contrast. The images show a single clump of filamenting cells, of which only the tips are in focus. The two brightly fluorescing patches shown in (a) correspond to the tips of the cell chains shown in (b). The samples were examined in a series of focal planes; fluorescence was only seen in the apical cells. Control L5366hpYES2 cells showed no detectable fluorescence (not shown).

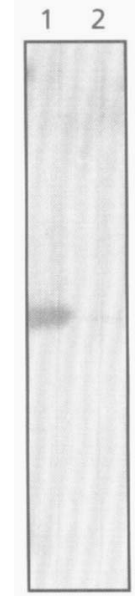

Fig. 7. Whi2 is recovered in the insoluble cell fraction. Insoluble (lane 1) and soluble (lane 2) protein fractions were prepared from W303a cells transformed with PWHIM7 induced as described in the legend to Fig. 1. Samples originating from equal amounts of cells were loaded onto an SDS gel and Western blotted and probed with 9E10 monoclonal antibody.

straightforward interpretation of this observation would place WHI2 upstream of STE11. However, the response is not blocked by ste 7 or ste 12 mutations which lie downstream of STE11 in both the mating pheromonetransduction pathway and pseudohypha formation (Liu et al., 1993). Either the response to Whi2 overproduction does involve the mating pheromone-response pathway but the order of function of its components is different from that seen previously, or STE11 has an additional function in another pathway.

There has been considerable interest recently in the mechanisms involved in bud site selection. BUD1 (also 


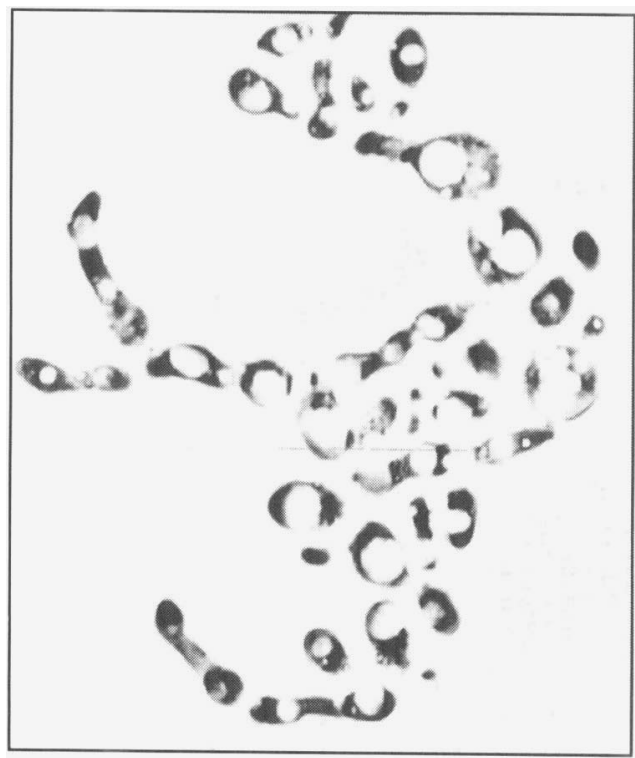

Fig. 8. Appearance of $f s w 1$ cells. Cells were grown overnight in liquid YEPD and examined with a phase-contrast microscope.

known as RSR1), BUD2 and BUD5 are required for both bipolar and axial budding patterns, since mutations lead to random bud-site selection (Bender \& Pringle, 1989; Chant \& Herskowitz, 1991; Chant et al., 1991). BUD3 $B U D 4$ and BUD10 are required specifically for the axial budding pattern, as mutations do not affect the bipolar budding pattern in a/ $\alpha$ diploids but cause $\mathbf{a}$ and $\alpha$ cells to bud in a bipolar fashion (Bender \& Pringle, 1989; Chant \& Herskowitz, 1991; Chant et al., 1991; Halme et al., 1996). A search for mutants specifically affected in the bipolar pattern yielded SPA2 (Zahner et al., 1996), a non-essential gene previously identified because mutant alleles result in an altered budding pattern and display weak defects in cytokinesis (Snyder, 1989). The search also yielded novel alleles of BUD2 and BUD5 and identified mutant alleles of four novel genes. Among these were bud6 and bud7 which budded randomly, bud8 which bud exclusively at the proximal pole and bud 9 which bud only at the distal pole. A recent model (Chant \& Pringle, 1995) for bud formation proposes that axial specific signals (Bud3, Bud4, etc.) and bipolarspecific signals both act upstream of general siteselection functions (Rsr1/Bud1, Bud2 Bud5). These act upon polarity establishment functions (Cdc24, Cdc42, Bem1) which in turn act upon the cytoskeleton. Overexpression of Whi2 modifies this pathway in at least three different ways: it changes the budding pattern from axial to unipolar, it results in hyperpolarized growth and it results in a failure to complete cytokinesis, which suggests that some essential function in budding has not been completed.

The change in budding pattern could result from down regulation or inactivation of BUD3 and/or BUD4 which would abrogate the axial budding pattern. It is unclear what causes the polarized growth. spa2 alleles are co-lethal with slk1, a component of the PKC1/MPK1 signalling pathway. We shall describe elsewhere extensive genetic interactions between WHI2 and this pathway and furthermore how whi2 cells have a disorganized actin cytoskeleton (unpublished). These observations suggest that both Whi2 and known components of the bud selection and formation pathways are part of a common system that interacts with the cytoskeleton. The cytokinesis defect resembles the phenotype of $c d c 3$, $c d c 10, c d c 11$ and $c d c 12$ alleles. These genes encode septins, which associate in a complex of $10 \mathrm{~nm}$ filaments that forms a ring defining the size and position of the bud neck at an early stage in bud formation, coincident with the assembly of the actin cap (reviewed by Sanders \& Field, 1994). cla mutants were isolated in a screen for mutations synthetically lethal in combination with $\operatorname{cln} 1$ and $\operatorname{cln} 2$ (Curckova et al., 1995). They also have a cytokinesis defect, and in fact cla10 is allelic to $c d c 12$. CLA4 encodes a kinase that is similar to Ste20 and the mammalian-brain-specific Pak kinase (Manser et al., 1994). cla4 is synthetically lethal in combination with ste 20 , but interestingly, not with ste 11 , ste $7, k s s 1$ or fus 1 , whose wild-type alleles encode members of a mitogenactivated protein kinase cascade which normally acts downstream of Ste20. Thus it is thought that Ste20 may participate in a second signalling pathway (c.f. the role of Ste11 in WHI2 overexpression). Since the cla mutants were uncovered in a synthetic lethal screen involving mutant alleles of CLN1 and CLN2, it has been suggested that both the Cln1/2-activated p34 kinase and the Cla4/Ste20 kinases are required to complete an event in bud formation. Thus the failure to complete cytokinesis is seen as a late consequence of an earlier defect.

We report elsewhere that, directly or indirectly, WHI2 regulates $\mathrm{G} 1$ cyclins since $w$ hi 2 cells have elevated levels of CLN1 and CLN2 transcripts which persist longer upon starvation than WHI2 cells (Radcliffe et al., 1997). There may thus be a connection between the observations reported here and the cla mutants - in both cases CLN activity is likely to be compromised and both result in a failure to complete cytokinesis. Characterization of the FSW1 gene, which is currently underway, will test these speculations.

\section{ACKNOWLEDGEMENTS}

P.A.R. and K.M.B. were in receipt of BBSRC Research Training Awards. We gratefully acknowledge strains provided by Dr H.-U. Mosch and Professor G. R. Fink. We thank Dr Martin Cambray-Deakin for assistance with the confocal microscopy.

\section{REFERENCES}

Adams, A. E. M. \& Pringle, J. R. (1983). Relationship of actin and tubulin distribution to bud growth in wild type and morphogenetic mutant Saccharomyces cerevisiae. J Cell Biol 98, 934-945. 
Bender, A. \& Pringle, J. R. (1989). Multicopy suppression of the $c d c 24$ budding defect in yeast by $C D C 42$ and 3 newly identified genes including the ras-related gene RSR1. Proc Natl Acad Sci USA 86, 9976-9980.

Chant, J. \& Herskowitz, I. (1991). Genetic control of bud site selection in yeast by a set of gene products that constitute a morphogenetic pathway. Cell 65, 1203-1212.

Chant, J. \& Pringle, J. R. (1995). Patterns of bud-site selection in the yeast Saccharomyces cerevisiae. J Cell Biol 129, 751-765.

Chant, J., Corrado, K., Pringle, J. R. \& Herskowitz, I. (1991). Yeast BUD5, encoding a putative GDP-GTP exchange factor, is necessary for bud site selection and interacts with bud formation gene BEM1. Cell 65, 1213-1224.

Crrckova, F., Devirgilio, C., Manser, E., Pringle, J. R. \& Nasmyth, K. (1995). Ste20-like protein-kinases are required for normal localization of cell-growth and for cytokinesis in budding yeast. Genes Dev 9, 1817-1830.

Elliott, B. \& Futcher, B. (1993). Stress resistance of yeast cells is largely independent of cell cycle phase. Yeast 9, 33-42.

Freifelder, D. (1960). Bud position in Saccharomyces cerevisiae. J Bacteriol 80, 567-568.

Gimeno, C. J. \& Fink, G. R. (1992). The logic of cell division in the life cycle of yeast. Science 257,626 .

Gimeno, C. J., Ljungdahl, P. O., Styles, C. A. \& Fink, G. R. (1992). Unipolar cell divisions in the yeast Saccharomyces cerevisiae lead to filamentous growth : regulation by starvation and RAS. Cell 68, 1077-1090.

Halme, A., Michelitch, M., Mitchell, E. L. \& Chant, J. (1996). Bud10p directs axial cell polarisation in budding yeast and resembles a transmembrane receptor. Curr Biol 6, 570-579.

Hartwell, L. H. (1974). The Saccharomyces cell cycle. Bacteriol Rev 38, 164-198.

Johnston, G. C., Pringle, J. R. \& Hartwell, L. H. (1977). Coordination of growth with cell division in the yeast Saccharomyces cerevisiae. Exp Cell Res 105, 79-98.

Kelly, D. E., Trevethick, J., Mountain, H. \& Sudbery, P. E. (1988). Transcript characterization, gene disruption and nucleotide sequence of the Saccharomyces cerevisiae WHI2 gene. Gene 66, 205-213.

Kurjan, J. (1993). The pheromone response pathway in Saccharomyces cerevisiae. Annu Rev Genet 27, 147-179.

Liu, H., Styles, C. A. \& Fink, G. R. (1993). Elements of the yeast pheromone response pathway required for filamentous growth of diploids. Science 262, 1741-1744.

Manser, E., Leung, T., Salihuddin, H., Zhao, Z. S. \& Lim, L. (1994). A brain serine specific protein kinase activated by $\mathrm{Cdc} 42$ and Rac1. Nature 367, 40-46.

Mountain, H. A. \& Sudbery, P. E. (1990a). Regulation of the Saccharomyces cerevisiae WHI2 gene. J Gen Microbiol 136, 727-732.

Mountain, H. A. \& Sudbery, P. E. (1990b). The relationship of growth rate and catabolite repression with WHI2 expression and cell size in Saccharomyces cerevisiae. J Gen Microbiol 136, 733-737.

Pringle, J. R. \& Hartwell, L. H. (1981). The Saccharomyces cerevisiae cell cycle. In The Molecular Biology of the Yeast Saccharomyces: Life Cycle and Inheritance, pp. 97-142. Edited by J. N. Strathern, E. W. Jones \& J. R. Broach. Cold Spring Harbor, NY: Cold Spring Harbor Laboratory.

Pringle, J. R., Adams, A. E. M., Drubin, D. G. \& Haarer, B. K. (1991). Immunofluorescence methods for yeast. In Guide to Yeast Genetics and Molecular Biology, pp. 565-601. Edited by C. Guthrie \& G. R. Fink. San Diego, CA : Academic Press.

Radcliffe, P. A., Binley, K. M., Trevethick, J. \& Sudbery, P. E. (1997). Deregulation of G1 cyclins in the Saccharomyces cerevisiae WHI2 mutant. Yeast (in press).

Rahman, D. R. J., Sudbery, P. E., Kelly, S. \& Marison, I. W. (1988) The effect of dissolved oxygen concentration on the growth physiology of Saccharomyces cerevisiae whi2 mutants. J Gen Microbiol 134, 2241-2248.

Reisdorf, P., Maarse, A. C. \& Daignan-Fournier, B. (1993). Epitope tagging vectors for yeast. Curr Genet 23, 181-183.

Roberts, R. L. \& Fink, G. R. (1994). Elements of a single MAP kinase cascade in Saccharomyces cerevisiae mediate two developmental programs in the small cell type: mating and invasive growth. Genes Dev 8, 2974-2985.

Sanders, S. L. \& Field, C. M. (1994). Cell division: septins in common? Curr Biol 4, 907-910.

Saul, D. J. \& Sudbery, P. E. (1985). Molecular-cloning of whi2, a gene involved in the regulation of cell-proliferation in Saccharomyces cerevisiae. J Gen Microbiol 131, 1797-1806.

Saul, D. J., Walton, E. F., Sudbery, P. E. \& Carter, B. L. A. (1985). Saccharomyces cerevisiae whi2 mutants in stationary phase retain the properties of exponentially growing cells. J Gen Microbiol 131, 2245-2251.

Snyder, M. (1989). The Spa2 protein of yeast localizes to sites of cell-growth. J Cell Biol 108, 1419-1429.

Sudbery, P. E., Goodey, A. R. \& Carter, B. L. C. (1980). Genes that control cell proliferation in the yeast Saccharomyces cerevisiae. Nature 288, 401-404.

Werner-Washburne, M., Braun, E., Johnston, G. C. \& Singer, R. A. (1993). Stationary phase in the yeast Saccharomyces cerevisiae. Microbiol Rev 57, 383-401.

Wright, R. M., Repine, T. \& Repine, J. E. (1993). Pseudohyphal growth in haploid Saccharomyces cerevisiae is a reversible process. Curr Genet 23, 388-391.

Zahner, J. E., Harkins, H. A. \& Pringle, J. R. (1996). Genetic analysis of the bipolar pattern of bud site selection in the yeast Saccharomyces cerevisiae. Mol Cell Biol 16, 1857-1870.

Received 14 October 1996; revised 8 January 1997; accepted 16 January 1997. 